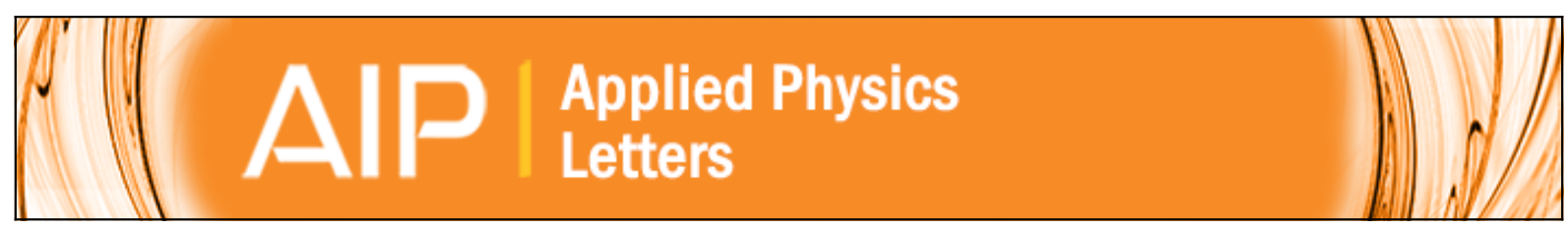

\title{
A phase field model for dislocation climb
}

Pierre-Antoine Geslin, Benoît Appolaire, and Alphonse Finel

Citation: Applied Physics Letters 104, 011903 (2014); doi: 10.1063/1.4860999

View online: http://dx.doi.org/10.1063/1.4860999

View Table of Contents: http://scitation.aip.org/content/aip/journal/apl/104/1?ver=pdfcov

Published by the AIP Publishing

$\stackrel{A}{A} \mathbb{P} P$ Re-register for Table of Content Alerts

Create a profile.

Sign up today! 


\title{
A phase field model for dislocation climb
}

\author{
Pierre-Antoine Geslin, ${ }^{\text {a) }}$ Benoît Appolaire, and Alphonse Finel \\ Laboratoire d'Etude des Microstructures, Onera/CNRS, 29 avenue de la division Leclerc, 92322 Châtillon, \\ France
}

(Received 24 September 2013; accepted 17 December 2013; published online 7 January 2014)

\begin{abstract}
We propose a phase field method to model consistently dislocation climb by vacancy absorption or emission. It automatically incorporates the exact balance between the vacancy flux and the phase field associated with the dislocation evolution, enforced by the conserved character of the total population of vacancies. One of its major advantage is the natural introduction of a dynamic coefficient controlling the kinetics of vacancy emission/absorption by the dislocation. We also derived a closed-form expression of the climb rate valid from the diffusion-limited to the attachment-limited regimes. (C) 2014 AIP Publishing LLC. [http://dx.doi.org/10.1063/1.4860999]
\end{abstract}

Dislocations are linear defects responsible for plastic deformation in crystalline solids. These defects change glide plane by absorbing point defects (climb). Whereas this climb mechanism is inhibited at low temperature, it becomes an essential part of plastic activity at high-temperature (creep). Therefore, the analysis of climb dynamics is mandatory to have a better understanding of the creep behavior of metals and alloys.

Kabir et al. ${ }^{1}$ used a Monte-Carlo method to study dislocation climb in iron. The vacancy concentration and the dislocation density in these simulations are inherently very high because of the limited system size. Climb has also been investigated qualitatively with the phase field crystal technique $^{2}$ but with the same limitation in system size.

To access more relevant scales, recent works ${ }^{3-5}$ introduced climb in Dislocation Dynamics (DD) modeling based on vacancy diffusion. The climb rate is obtained by integrating analytically Fick's equation with strong assumptions. Especially, each point of the dislocation is assumed to act as a perfect source/sink of vacancies and the elastic interactions between dislocations and vacancies are neglected. This first assumption can be spurious in some cases where the jogs concentration along the line is low and climb becomes limited by point defect emission/absorption. ${ }^{6}$

Gao et $a l^{7}$ coupled pipe diffusion theory and DD to investigate climb dynamics in case of low jog concentration, but neglecting bulk diffusion, an essential feature of dislocation climb. Among the previously developed models, none is able to investigate climb behavior influenced by both bulk diffusion and absorption/emission kinetics.

The phase field method seems particularly adapted to the study of dislocation climb because it naturally incorporates diffusion processes. Phase field models have been extensively used to reproduce microstructure evolutions at mesoscale. ${ }^{8,9}$ They rely on an out-of-equilibrium thermodynamics formulation, incorporate elastic long range interactions and the coupling of a microstructure with defects, such as dislocations. ${ }^{10-13}$ It is therefore essential to extend its applicability to dislocation climb. A phase field approach of climb has been proposed recently ${ }^{14}$ to analyze prismatic loop

a)pageslin@onera.fr evolution by point defect absorption in irradiated iron. However, this formulation does not guarantee the correct balance between the defect fluxes in the matrix and the dislocation evolution (the dislocation loop may evolve unrealistically without defect fluxes in the matrix).

In the present Letter, we propose a general phase field model to investigate dislocation climb. We distinguish bulk vacancies and vacancies composing dislocation loops and formulate dynamic equations such that the total number of vacancies is conserved. By means of a dynamic coefficient, the exchange rate between the two populations of vacancies can be controlled. This parameter can account for a limiting absorption/emission mechanism. We study its influence on the climb rate.

We restrict ourselves to the case of non-irradiated materials where the role of interstitials on climb is negligible compared to vacancies. Our model relies on different fields, the bulk vacancy concentration denoted by $c(r)$ and a set of phase fields $\phi_{i}(r)$ representing dislocation loops lying within specific crystallographic planes: $\phi_{i}(r)=1$ if $r$ is inside a loop of type $i$ and 0 outside. For the sake of simplicity, we will focus on a single loop within a single plane and along which the dislocation is purely edge. Generalization to more complex situations is straightforward.

The free energy density of the system is decomposed in three terms, the vacancy free energy $f_{c h}(c)$, the dislocation core energy $f_{\text {core }}(\phi)$, and the elastic energy $f_{e l}(\phi, c)$.

In the dilute solution limit, we have

$$
f_{c h}(c)=\frac{E_{f}}{\Omega} c+\frac{k T}{\Omega}[c \ln (c)+(1-c) \ln (1-c)],
$$

where $E_{f}$ is the formation energy of vacancies and $\Omega$ the atomic volume.

The dislocation core energy $f_{\text {core }}(\phi)$ is written as the sum of a double-well potential and a gradient term

$$
f_{\text {core }}(\phi)=A \phi^{2}(1-\phi)^{2}+\frac{B}{2}|\boldsymbol{n} \wedge \nabla \phi|^{2}
$$

where $\boldsymbol{n}$ denotes the normal to the vacancy loop. To avoid unrealistic surface energy on the surface of the loop, the gradient term contribution is nullified along $\boldsymbol{n}$. The width and 
energy of the dislocation core determine the parameters $A$ and $B$.

The elastic energy is expressed as

$$
f_{e l}(c, \phi, \boldsymbol{\epsilon})=\frac{1}{2}\left(\boldsymbol{\epsilon}-\boldsymbol{\epsilon}_{\mathbf{0}}(c, \phi)\right): \boldsymbol{C}:\left(\boldsymbol{\epsilon}-\boldsymbol{\epsilon}_{\mathbf{0}}(c, \phi)\right)-\boldsymbol{\sigma}^{\boldsymbol{a}}: \boldsymbol{\epsilon},
$$

where $\boldsymbol{C}$ is the stiffness tensor, $\boldsymbol{\sigma}^{\boldsymbol{a}}$ the applied stress tensor, and double dots ":" denote double contractions. The eigenstrain tensor $\boldsymbol{\epsilon}^{\mathbf{0}}(c, \phi)$ can be divided into a vacancy contribution $\boldsymbol{\epsilon}_{v}^{\mathbf{0}}(c)$ and a dislocation loop contribution $\boldsymbol{\epsilon}_{l}^{\mathbf{0}}(\phi)$. The eigenstrain contribution of a single loop is expressed as follows: $\varepsilon_{l, i j}^{0}(\phi)=\frac{1}{2}\left(b_{i} n_{j}+b_{j} n_{i}\right) h(\phi) \delta(\xi)$, where $b_{i}$ is the component of the dislocation Burgers vector, $\delta$ the Dirac function, and $\xi$ the distance to the loop plane. The function $h(\phi)$ is a monotonic function with $h(0)=0, h(1)=1$, and $h^{\prime}(0)=h^{\prime}(1)=0$. The generalization to any number of loops is straightforward. We suppose that the vacancy eigenstrain varies linearly with $c$, which is valid for low concentrations, and that the eigenstrain associated with a single vacancy is $\delta_{i j} V^{*} / 3 \Omega\left(V^{*}\right.$ is the vacancy relaxation volume). In this paper, we neglect the influence of elastic interaction between vacancies and dislocations by setting $V^{*}=0$. A detailed study on the influence of this parameter on the climb rate will be presented elsewhere.

The phase field $\{\phi\}$ and the vacancy field $\{c\}$ are not conserved. However, the dislocation loop grows/shrinks by absorbing/emitting vacancies such that the combined field $\psi=c+\phi$ is conserved. Therefore, to ensure its conservation, the evolution of $\{\psi\}$ is supposed to follow a CahnHilliard equation:

$$
\dot{\psi}=\nabla \cdot M \nabla \frac{\delta F^{*}}{\delta \psi}
$$

where the driving force is controlled by the total free energy $F^{*}(\{\psi\},\{\phi\},\{\boldsymbol{\epsilon}\})$ expressed in terms of the fields $\{\psi\}$ and $\{\phi\}$ and $\{\boldsymbol{\epsilon}\}$ (kinetics on strain fields $\{\boldsymbol{\epsilon}\}$ will be addressed below). The physical meaning of the mobility $M$ will be discussed below. To close the set of dynamical equations, we write an Allen-Cahn dynamics on the non-conserved parameter $\{\phi\}$

$$
\dot{\phi}=-L \frac{\delta F^{*}}{\delta \phi}
$$

where $L$ is a kinetic coefficient. In order to express the dynamics in terms of the more natural concentration and dislocation fields $\{c\},\{\phi\}$, the change of variable $c=\psi-\phi$ is performed to give

$$
\begin{gathered}
\dot{c}=\nabla \cdot M \nabla \frac{\delta F}{\delta c}+L\left(\frac{\delta F}{\delta \phi}-\frac{\delta F}{\delta c}\right), \\
\dot{\phi}=-L\left(\frac{\delta F}{\delta \phi}-\frac{\delta F}{\delta c}\right),
\end{gathered}
$$

where now the free energy $F(\{c\},\{\phi\},\{\boldsymbol{\epsilon}\})$ is a functional of the fields $\{c\}$ and $\{\phi\}$ and is expressed as the integral of the sum of the densities of Eqs. (1)-(3). A quick inspection of Eq. (6) indicates that $M$ is simply the vacancy mobility, related to the diffusivity $D_{v}$ by $M(c)=\Omega D_{v} c(1-c) / k T$ for dilute systems. The term $L(\delta F / \delta \phi-\delta F / \delta c)$ plays the role of a source term in Eq. (6) and controls the exchanges between the fields $\{\phi\}$ and $\{c\}$ at a rate related to $L$.

The absorption/emission of vacancies at the dislocation core is usually assumed to be fast compared to the bulk diffusion of vacancies, ${ }^{3,15}$ such that the surrounding vacancies remain at equilibrium with the dislocation cores. This is the local equilibrium assumption. However, the vacancy absorption/emission at the dislocation is a complex mechanism involving the jog density along the line, pipe diffusion, and the elementary mechanism of absorption/emission at a jog. In fcc and hep metals, jogs are high energy defects and their concentration along the dislocation can be low. ${ }^{6}$ In such cases, the absorption/emission mechanism involves the diffusion of point defects to/from the existing jogs and the nucleation of new jogs, two phenomena slowing down the dislocation climb. Analytical models for the climb rate in these situations can be found in the literature. ${ }^{6,16,17}$ The existence of such regimes where the climb rate is limited by the absorption mechanism is supported by experimental works: on gold $^{18}$ where the measured climb rate differs from the one predicted with the local equilibrium assumption, and on magnesium ${ }^{6,19}$ where the activation energy of climb is found to be significantly larger than the self-diffusion energy.

The parameter $L$ can be chosen to reproduce the whole spectrum of behaviors between these two limit cases. In the spirit of a coarse-graining procedure, the parameter $L$ reflects a lower space and time scale kinetic process which appears at mesoscale as a thermally activated mechanism. Therefore, $L$ should be written as $L=L_{0} \exp (-\Delta E / k T)$, where $\Delta E$ is an energy barrier (possibly involving the jog formation energy) and $L_{0}$ a coefficient independent of temperature.

The dimensionless equations are obtained after normalization with the characteristic length $l_{0}=b$, time $t_{0}=b^{2} / D_{v}$, and energy $E_{0}=k T b^{3} / \Omega c_{0}\left(1-c_{0}\right)$, where $c_{0}$ is the equilibrium vacancy concentration. In particular, the dimensionless vacancy mobility at equilibrium becomes $\bar{M}\left(c_{0}\right)=1$ and the dimensionless kinetic coefficient is written as $\bar{L}=L b^{2} / M\left(c_{0}\right)$. Thus, if $\bar{L} \gg 1$ (respectively, $\bar{L} \ll 1$ ), the attachment dynamics is much faster (much slower) than vacancy bulk diffusion.

Equations (6) and (7) are integrated numerically with a finite difference method on a staggered grid $^{20,21}$ in Fourier space and using an explicit Euler scheme. As elastic relaxation is much faster than diffusion processes, the elastic fields are considered to follow quasi-statically the concentration and plastic fields $c$ and $\phi$. Therefore, static mechanical equilibrium $\nabla \cdot \boldsymbol{\sigma}=0$ is solved at each time step.

We use the parameters of aluminum (see Table I) taken from Refs. 22 and 23. We use the Voigt isotropic average ${ }^{22}$ under the argument that elastic anisotropy is small in aluminum. The dislocation core energy has been arbitrary set to $E_{c}=1.0 \mathrm{eVA}^{-1}$.

The equations are discretized on a grid of dimensions $L_{x}=364.8 \AA$ and $L_{y}=729.60 \AA$ with a grid spacing $d=2.85 \AA$. A dislocation dipole is introduced with a platelet $\phi=1$ of thickness $d$ at the center of the simulation box (see inset in Fig. 1). Elastic equilibrium and Eq. (7) are solved with periodic boundary conditions whereas the vacancy 
TABLE I. Simulation parameters. ${ }^{22,23}$

\begin{tabular}{lc}
\hline \hline Burgers vector $b$ & $2.85 \AA$ \\
Elastic constant $\lambda$ & $59 \mathrm{GPa}$ \\
Elastic constant $\mu$ & $26 \mathrm{GPa}$ \\
Vacancy formation energy $E_{f}$ & $0.67 \mathrm{eV}$ \\
Vacancy migration energy $E_{m}$ & $0.61 \mathrm{eV}$ \\
Pre-exponential factor $D_{0}$ & $1.51 \times 10^{-5} \mathrm{~m}^{2} \mathrm{~s}^{-1}$ \\
Atomic volume $\Omega$ & $16.4 \AA^{3}$ \\
Dislocation core energy $E_{c}$ & $1.0 \mathrm{eV} \AA^{-1}$ \\
Dislocation core width $w_{c}$ & $17.1 \AA$ \\
\hline \hline
\end{tabular}

equilibrium concentration $c_{0}$ is imposed at the boundaries to mimic an infinite source. Different external shear stresses $\sigma^{a}$ (inset in Fig. 1) are applied to study the influence of the mechanical force on the climb rate. The climb rate is determined once a stationary regime is reached and before elastic interactions with periodic images become significant.

Fig. 1 displays the stationary climb rate (symbols) as a function of the applied stress for different values of the kinetic absorption/emission coefficient $\bar{L}$.

As observed in Fig. 1, the decrease of $\bar{L}$ changes drastically the climb rate behavior. This behavior can be investigated further by means of an analytical treatment (asymptotic analysis) in a stationary regime. We assume that the characteristic length of the vacancy concentration profile is much larger than the core width. In this limit, we expect that the phase field will be of the form $\phi(r, t)=\phi\left(r-R_{0}(t)\right)$, where $R_{0}(t)$ represents the position of the dislocation core. The spatial derivative of $\phi$ along the climb direction is sharply peaked around the dislocation position on a length scale of the order of the dislocation core. Multiplying Eq. (7) by this derivative and integrating through a small domain leads to a single equation that relates the climb rate $v_{1}=\dot{R}_{0}$ to the concentration $c_{d}$ in the neighborhood of the core and to the applied stress $\sigma_{a}$

$$
v_{1}=\frac{3 L w}{2}\left(\mu\left(c_{d}\right)-\sigma^{a}\right),
$$

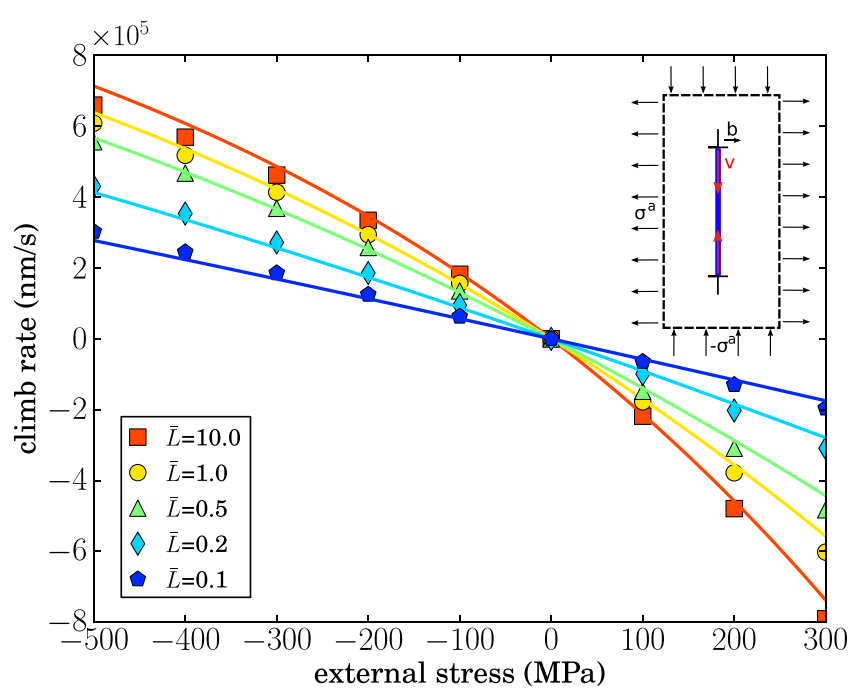

FIG. 1. Climb rate vs. external stress $\sigma_{a}$ for different values of $\bar{L}$ at $T=812 \mathrm{~K}$ from simulation results (symbols) and analytical solutions for the climb rate combining Eqs. (9) and (10) with $\eta=2 \pi / \ln \left(R / r_{d}\right)$ as a fitting parameter (lines). Simulation set up (inset). where $\mu\left(c_{d}\right)=\partial f_{c h} / \partial c$ denotes the chemical potential and $w$ the core width. To find the concentration $c_{d}$ which depends on the vacancy concentration profile, we consider that the vacancies follow a quasi-static kinetics with respect to the dislocation field. This is a valid approximation in the case of a moderate driving force. ${ }^{24}$ By approximating the chemical potential by $\mu(c)=k T \ln \left(c / c_{0}\right)$, the stationary equation for the concentration $c$ becomes $\Delta c=0$. Following standard approaches, ${ }^{3,22}$ this equation can be solved in a hollow cylinder centered on the dislocation with external radius $R$ and internal radius $r_{d}$ subjected to the boundary conditions $c(R)=c_{0}$ and $c\left(r_{d}\right)=c_{d}$. The climb rate is then obtained from the spatially averaged vacancy flux towards the dislocation

$$
v_{2}=\frac{2 \pi D_{v} c_{0}}{b \ln \left(R / r_{d}\right)}\left(1-\frac{c_{d}}{c_{0}}\right)
$$

which is also function of $c_{d}$. The climb rates given by Eqs. (8) and (9) should of course be the same. This leads to a boundary condition on $c_{d}$ which reads

$$
\frac{c_{d}}{c_{0}}=\frac{\bar{L}}{\Gamma} W\left(\frac{\Gamma}{\bar{L}} \exp \left(\frac{\Gamma}{\bar{L}}+\frac{\sigma^{a} \Omega}{k T}\right)\right),
$$

where $W(x)$ is the Lambert function, defined by $W(x) e^{W(x)}=x$ and $\Gamma=4 \pi b / 3 w \ln \left(R / r_{d}\right)$ is a dimensionless factor function of geometrical parameters and is of the order of unity. Injecting this solution in Eq. (9) leads to the dislocation climb rate. In the limit $\bar{L} \gg \Gamma$, where the climb rate is controlled by diffusion, a first order expansion in $\Gamma / \bar{L}$ leads to

$$
v_{\bar{L} \gg \Gamma} \simeq \frac{2 \pi D_{v} c_{0}}{b \ln \left(R / r_{d}\right)}\left[1-e^{\frac{\sigma^{a} \Omega}{k T}}\left(1+\frac{\Gamma}{\bar{L}}\left(1-e^{\frac{\sigma^{a} \Omega}{k T}}\right)\right)\right] .
$$

When $\bar{L} \rightarrow+\infty$, we obtain exactly the solution derived with the local equilibrium assumption. ${ }^{3,22}$ In the opposite case $\bar{L} \ll \Gamma$, where climb is controlled by the emission/absorption kinetics, an asymptotic expansion of the $W$-Lambert function gives

$$
v_{\bar{L} \ll \Gamma} \simeq-\frac{3}{2} w \sigma^{a} L,
$$

where the expressions of $\bar{L}$ and $\Gamma$ have been used. In this limit, the climb rate is linear in $\sigma^{a}$ and $L$, as expected.

To compare quantitatively the numerical climb rate with the analytical solution, a least-square fit is performed on our numerical results with the geometrical factor $\eta=2 \pi / \ln \left(R / r_{d}\right)$ as the only fitting parameter. Indeed, this parameter may differ from its analytical expression considering that the boundary condition in the numerical and analytical solutions are not the same. We find $\eta=2.3$ which is still very close to $2 \pi / \ln \left(L_{x} / w\right)=2.1$, obtained with the natural choices for the external radius $R=L_{x} / 2$ and the internal radius $r_{d}=w / 2$. The continuous lines in Fig. 1 represent the fitted solution. The small discrepancies may come from the elastic interactions between periodic images in the numerical results and from the non-stationarity of the concentration field naturally incorporated in our simulation and neglected in the analytical treatment.

In conclusion, we have proposed a phase field model for dislocation climb. A dynamical coefficient enables to control 
the kinetic of emission/absorption of vacancies at the dislocation. Using this coefficient, the whole spectrum between absorption-limited and diffusion-limited climb is accessible. An analytical analysis of the model is presented and a closed-form expression of the climb rate is derived, which reproduces with accuracy the numerical data obtained with our simulations. In principle, a quantitative estimate of the parameter $L$ can be obtained from large scale molecular dynamics simulations. It can also be evaluated by analyzing vacancy diffusion profile at the level of the jogs with an analytical approach similar to the asymptotic analysis used above. This would lead to an expression of an effective dynamic coefficient $L$ function of dislocation properties (jog density, pipe diffusion coefficient). ${ }^{25}$ Large scale simulations, such as those needed to study creep can be reached within this phase field approach by using a larger grid spacing, provided it stays smaller than any other characteristic length scales (in particular dislocations curvature radius and mean dislocation spacing). This phase field model also opens the possibility to investigate the climb behavior of dislocations junctions, and walls, out of reach of the current DD approaches $^{3-5}$ because of the small distances between dislocations. Moreover, this model can be coupled to a phase field model for dislocation glide ${ }^{11}$ and will thus provide a complete picture of dislocation dynamics in solids and its coupling to microstructure evolutions.

We would like to thank Yann Le Bouar for fruitful discussions.
${ }^{1}$ M. Kabir, T. Lau, D. Rodney, S. Yip, and K. Van Vliet, Phys. Rev. Lett. 105, 095501 (2010).

${ }^{2}$ J. Berry, M. Grant, and K. Elder, Phys. Rev. E 73, 031609 (2006).

${ }^{3}$ D. Mordehai, E. Clouet, M. Fivel, and M. Verdier, Philos. Mag. 88, 899 (2008).

${ }^{4}$ B. Bakó, E. Clouet, L. M. Dupuy, and M. Blétry, Philos. Mag. 91, 3173 (2011). ${ }^{5}$ S. Keralavarma, T. Cagin, A. Arsenlis, and A. Benzerga, Phys. Rev. Lett. 109, 265504 (2012).

${ }^{6}$ D. Caillard and J.-L. Martin, Thermally Activated Mechanisms in Crystal Plasticity (Pergamon Press, Amsterdam, 2003), Chap. VIII.

${ }^{7}$ Y. Gao, Z. Zhuang, Z. Liu, X. You, X. Zhao, and Z. Zhang, Int. J. Plasticity 27, 1055 (2011).

${ }^{8}$ L. Chen, Annu. Rev. Mater. Res. 32, 113 (2002).

${ }^{9}$ G. Boussinot, Y. Le Bouar, and A. Finel, Acta Mater. 58, 4170 (2010).

${ }^{10} \mathrm{D}$. Rodney and A. Finel, "Phase field methods and dislocations," MRS Proc. 652, Y4.9 (2000).

${ }^{11}$ D. Rodney, Y. Le Bouar, and A. Finel, Acta Mater. 51, 17 (2003).

${ }^{12}$ Y. Wang, Y. Jin, and A. Cuitino, Acta Mater. 49, 1847 (2001).

${ }^{13}$ V. Vorontsov, C. Shen, Y. Wang, D. Dye, and C. Rae, Acta Mater. 58, 4110 (2010).

${ }^{14}$ Y. Li, S. Hu, C. Henager, H. Deng, F. Gao, X. Sun, and M. Khaleel, J. Nucl. Mater. 427, 259 (2012).

${ }^{15}$ J. Lothe and J. Hirth, J. Appl. Phys. 38, 845 (1967).

${ }^{16}$ J. Lothe, J. Appl. Phys. 31, 1077 (1960).

${ }^{17}$ R. Balluffi, Phys. Stat. Sol. B 31, 443 (1969).

${ }^{18}$ D. Seidman and R. Balluffi, Phys. Rev. 139, A1824 (1965).

${ }^{19}$ G. Edelin and J. Poirier, Philos. Mag. 28, 1211 (1973).

${ }^{20}$ E. Saenger, N. Gold, and S. Shapiro, Wave Motion 31, 77 (2000).

${ }^{21}$ J. Carcione, G. Herman, and A. Ten Kroode, Geophysics 67, 1304 (2002).

${ }^{22}$ J. Hirth and J. Lothe, Theory of Dislocations (McGraw-Hill, New-York, 1968).

${ }^{23}$ Landolt-Börnstein: Numerical Data and Functional Relationship in Science and Technology, edited by O. Madelung (Springer-Verleg, Berlin, 1990), Vol. 26

${ }^{24}$ R. Balluffi and D. Seidman, J. Appl. Phys. 36, 2708 (1965).

${ }^{25}$ P. A. Geslin, B. Appolaire, and A. Finel, "Climb of jogged dislocations: Analytical approach and upscaling to a mesoscopic phase field method" (unpublished). 